PROCEEDINGS OF THE

AMERICAN MATHEMATICAL SOCIETY

Volume 127, Number 3, March 1999, Pages 791-800

S 0002-9939(99)04586-4

\title{
JACOBI MATRICES WITH ABSOLUTELY CONTINUOUS SPECTRUM
}

\author{
JAN JANAS AND SERGUEI NABOKO
}

(Communicated by Palle E. T. Jorgensen)

\begin{abstract}
Let $J$ be a Jacobi matrix defined in $l^{2}$ as $R e W$, where $W$ is a unilateral weighted shift with nonzero weights $\lambda_{k}$ such that $\lim _{k} \lambda_{k}=1$. Define the seqences: $\varepsilon_{k}:=\frac{\lambda_{k-1}}{\lambda_{k}}-1, \delta_{k}:=\frac{\lambda_{k}-1}{\lambda_{k}}, \eta_{k}:=2 \delta_{k}+\varepsilon_{k}$. If $\varepsilon_{k}=$ $O\left(k^{-\alpha}\right), \eta_{k}=O\left(k^{-\gamma}\right), \frac{2}{3}<\alpha \leq \gamma, \alpha+\gamma>3 / 2$ and $\gamma>3 / 4$, then $J$ has an absolutely continuous spectrum covering $(-2,2)$. Moreover, the asymptotics of the solution $J u=\lambda u, \lambda \in \mathbb{R}$ is also given.
\end{abstract}

\section{INTRODUCTION}

Let $W$ be a unilateral weighted shift defined in $l^{2}$ by the formula $W e_{n}=$ $2 \lambda_{n} e_{n+1}$, for some real sequence $\left\{\lambda_{n}\right\}$. In this paper we consider the operator $J=\operatorname{Re} W$ which has the matrix form given by

$$
J=\left(\begin{array}{ccccc}
0 & \lambda_{1} & 0 & 0 & \cdots \\
\lambda_{1} & 0 & \lambda_{2} & 0 & \cdots \\
0 & \lambda_{2} & 0 & \lambda_{3} & \cdots \\
\vdots & \vdots & \vdots & &
\end{array}\right)
$$

The matrix structure of operator $J$ will be used to obtain information about absolutely continuous component of its spectrum. In what follows we always assume that $\lambda_{k} \neq 0$, for all $k$ and moreover that $\lim _{k} \lambda_{k}=1$. Then $J$ is a compact perturbation of $2 \operatorname{Re} U$, where $U$ is the unilateral shift, $U e_{n}=e_{n+1}$. In other words $J$ is a compact perturbation of a discrete Schrödinger operator with zero potential, i.e. $\lambda_{k}=1$. One can call this subclass of Jacobi matrices discrete string operators by analogy with the continuous case; see (2.1). Preservation of the absolutely continuous spectrum of $\operatorname{Re} U$ under certain assumptions on the rate of convergence of $\left\{\lambda_{k}\right\}$ to 1 will be given.

The methods used in the analysis of the above problem combine the standard theory of subordinacy due to Gilbert-Pearson [5], a slight modification of the HarrisLutz method [6] and a recent Kiselev approach to absolute continuity of discrete Schrödinger operators [9].

Received by the editors June 25, 1997.

1991 Mathematics Subject Classification. Primary 47B37; Secondary $47 B 39$.

Key words and phrases. Jacobi matrix, absolutely continuous spectrum, asymptotics behaviour.

The research of the first author was supported by grant PB 2 PO3A 00213 of the Komitet Badań Naukowych, Warsaw.

(C)1999 American Mathematical Society 
Let us explain briefly how these techniques and notions will be used below. Consider the system of equations

$$
\lambda_{n-1} u_{n-1}+\lambda_{n} u_{n+1}=\lambda u_{n}, \quad n>1,
$$

for a generalized solution eigenfunction $u=\left(u_{n}\right)_{n=2}^{\infty}$ which does not belong to $l^{2}$ in general [1]. Following [5] we call $u \neq 0$ a subordinate solution of (1.1) if for every solution $v$ of (1.1) which is not a constant multiple of $u$ we have

$$
\lim _{n \rightarrow \infty} \frac{\sum_{n=1}^{n}\left|u_{k}\right|^{2}}{\sum_{k=1}^{n}\left|v_{k}\right|^{2}}=0 .
$$

As a consequence of the Gilbert-Pearson theory we know that if for almost all $\lambda \in(a, b)$ there is no subordinate solution of $(1.1)$, then $(a, b) \subset \sigma(J)$ and the spectrum of $J$ has absolutely continuous component filling $(a, b)$. It is well known that boundedness of all solutions of (1.1) implies that no solution of (1.1) is subordinate [12]. Therefore the only point remaining concerns finding when all solutions of (1.1) are bounded. This point is analysed by applying a modified Harris-Lutz transform which linearly changes the coordinates and replaces the equation (1.1) by an equivalent one. The same strategy has been successfully used by Kiselev in his work [9] devoted to one-dimensional Schrödinger operator (discrete or continuous) with absolutely continuous spectrum.

One also could consider more general tridiagonal matrices with nonzero main diagonal (discrete potential) by combining our approach with the above mentioned Kiselev's results. We concentrate on the discrete string operator however, because its analysis brings new algebraic and analytic difficulties in comparison with the Discrete Schrödinger Operator (DSO). This is related to a more complicated algebraic structure of $J$; see formula (2.7) for the transfer matrix. For earlier works devoted or related to the question we consider in this paper we refer the reader to [2], [4], [7], [8], [11], [13]. The authors thank A. Kiselev for sending them his papers prior to publication.

\section{Preliminaries}

Let $\left\{e_{n}\right\}_{n=1}^{\infty}$ be the standard orthonormal basis in $l^{2}$. For a bounded sequence $\left\{\lambda_{k}\right\}$ of positive numbers denote by $\Lambda$ the diagonal operator given by $\Lambda e_{n}=\lambda_{n} e_{n}$. If $U$ is the unilateral shift, $U e_{n}=e_{n+1}$, then the operator

$$
J=(U \Lambda)^{*}+U \Lambda .
$$

Here we always assume that $\lim _{k} \lambda_{k}=1$. We introduce the new parameters $\varepsilon_{n}, \delta_{n}$ by

$$
\frac{\lambda_{n-1}}{\lambda_{n}}=1+\varepsilon_{n}, \quad 1-\frac{1}{\lambda_{n}}=\delta_{n} .
$$

Then one can easily check that

$$
\varepsilon_{k}=\delta_{k-1}-\delta_{k}+O\left(\delta_{k-1}\left(\delta_{k-1}-\delta_{k}\right)\right) k \geq 2 .
$$

The next parameter which will naturally appear later (see (2.8)) is:

$$
\eta_{k}:=2 \delta_{k}+\varepsilon_{k} .
$$

We have the relation

$$
\eta_{k}=\delta_{k-1}+\delta_{k}+O\left(\delta_{k-1}\left(\delta_{k-1}-\delta_{k}\right)\right) .
$$


In what follows we always assume that the parameters $\varepsilon_{n}, \delta_{n}$, and $\eta_{n}$ satisfy the following growth conditions:

$$
\varepsilon_{n}=O\left(n^{-\alpha}\right), \alpha>0, \quad \delta_{n}=O\left(n^{-\beta}\right), \beta>\frac{1}{2}, \quad \text { and } \quad \eta_{n}=O\left(n^{-\gamma}\right), \gamma>0 .
$$

By (2.3) and (2.5) one can easily check that

$$
\beta \geq \min (\alpha, \gamma) \text { and } \quad \alpha \geq \beta \leq \gamma .
$$

We have two possibilities:

(a) if $\min (\alpha, \gamma)=\alpha$, then $\alpha=\beta$,

(b) if $\min (\alpha, \gamma)=\gamma$, then $\alpha \geq \beta \geq \gamma \geq \beta$ and so $\beta=\gamma$.

Therefore we only have two independent parameters $\alpha$ and $\gamma$.

As we mentioned in the Introduction, Kiselev's results (the discrete Kiselev Lemma) will be used in the analysis of absolute continuity of $J$. Therefore for convenience to the readers we formulate this lemma.

Lemma (Kisielev). If a sequence $\left\{r_{n}\right\}$ belongs to $l^{2}$, then

$$
\sum_{k=1}^{N} r_{k} e^{i k \Theta}=O(\log N)
$$

for almost all $\Theta \in(-\pi, \pi)$.

The next result is a slight extension of Lemma 3.4 in [9]. Actually it was proved by Kiselev for $\rho=1 / 4$. Formally it is not contained in [9], however.

Lemma 2.1. For any complex sequence $\left\{q_{k}\right\}$ such that $\left\{q_{k} k^{\rho}\right\}$ is square summable for some $\rho>0$ we have

$$
\left|\sum_{k=n}^{\infty} q_{k} e^{-i k \Theta}\right|=O\left(n^{-\rho} \ln n\right),
$$

for almost all $\Theta \in(-\pi, \pi)$.

Proof. Applying the Abel summation formula we have

$$
\begin{gathered}
\sum_{k=n+1}^{\infty} q_{k} e^{-i k \Theta}=n^{-\rho} \sum_{k=n+1}^{\infty} q_{k} k^{\rho} e^{-i k \Theta} \\
+\sum_{k=n+1}^{\infty}\left[k^{-\rho}-(k-1)^{-\rho}\right] \sum_{j=k}^{\infty} q_{j} j^{\rho} e^{-i j \Theta}=O\left(n^{-\rho} \log n\right) .
\end{gathered}
$$

In the last equality, Kiselev's lemma and the Carleson theorem on almost everywhere convergence of Fourier series with square summable coefficients have been used.

In particular we have

Corollary 2.2. If $\varepsilon_{k}=O\left(k^{-\alpha}\right), \alpha>\frac{1}{2}$, then

$$
\left|\sum_{k=n}^{\infty} \varepsilon_{k} e^{i k \Theta}\right|=O\left(k^{-\alpha+\frac{1}{2}+\omega}\right), \quad \text { for all } \omega>0
$$

and almost all $\Theta \in(-\pi, \pi)$. 
The next result can be called a Levinson type theorem and will also be needed below.

Lemma 2.3. Let $B_{n}$ be a sequence of $s \times s$ matrices such that $\left\{\left\|B_{n}\right\|\right\} \in l^{1}$. Then for a sequence $A_{n}$ of $s \times s$ matrices the product $\prod_{k=1}^{n}\left(I+A_{k}+B_{k}\right)$ and its inverse is uniformly bounded from above in norm provided that the product

$\prod_{k=1}^{n}\left(I+A_{k}\right)$ has the same property.

We do not prove this simple result because its proof is very close to the continuous case, see [3, Chap.III, Exer.4].

For $\lambda \in(-2,2)$ consider a solution $u$ of the equation (1.1).

Let

$$
B_{n}:=\left(\begin{array}{cc}
0, & 1 \\
-\frac{\lambda_{n-1}}{\lambda_{n}}, & \frac{\lambda}{\lambda_{n}}
\end{array}\right)
$$

be the transfer matrix, i.e.

$$
\left(\begin{array}{c}
u_{n} \\
u_{n+1}
\end{array}\right)=B_{n}\left(\begin{array}{c}
u_{n-1} \\
u_{n}
\end{array}\right), n \geq 2
$$

Applying Stolz result [12, Cor.2] we look for criteria (restrictions on $\alpha$ and $\gamma$ ) which guarantee that $\left\{u_{n}\right\}$ is bounded. Actually his theorem was proved only for DSO but the same proof also works in our case.

Let $\lambda=2 \cos \Theta$. Denote

$$
\mu=\frac{\lambda}{2}+i \sqrt{1-\frac{\lambda^{2}}{4}}=\cos \Theta+i \sin \Theta .
$$

Note that the matrix $W=\left(\begin{array}{cc}1 & 0 \\ \operatorname{Re} \mu & \operatorname{Im} \mu\end{array}\right)$ is invertible and

$$
B_{n}^{\prime}:=W^{-1} B_{n} W=\left(I-\left(\begin{array}{cc}
O & O \\
\eta_{n} \operatorname{ctg} \Theta & -\varepsilon_{n}
\end{array}\right)\right) P
$$

where $P=\exp \left[\Theta\left(\begin{array}{cc}0 & 1 \\ -1 & 0\end{array}\right)\right]$ is the rotation matrix.

Let

$$
U=\frac{1}{2}\left(\begin{array}{cc}
1 & -i \\
1 & i
\end{array}\right)
$$

We have $P=U^{-1} e^{i \Theta B} U$, where $B=\left(\begin{array}{cc}1 & 0 \\ 0 & -1\end{array}\right)$.

The matrix $B_{n}^{\prime}$ can be expressed as follows:

$$
U^{-1}\left[\left(1+\frac{\varepsilon_{n}}{2}\right) I-\frac{\varepsilon_{n}}{2}\left(\begin{array}{ll}
0 & 1 \\
1 & 0
\end{array}\right)+\frac{i \eta_{n}}{2} \operatorname{ctg} \Theta\left(B+\left(\begin{array}{cc}
0 & 1 \\
-1 & 0
\end{array}\right)\right)\right] e^{i \Theta B} U .
$$

Denote by

$$
\tilde{B}_{n}:=\left[\left(1+\frac{\varepsilon_{n}}{2}\right) I-\frac{\varepsilon_{n}}{2}\left(\begin{array}{ll}
0 & 1 \\
1 & 0
\end{array}\right)+\frac{i \eta_{n}}{2} \operatorname{ctg} \Theta\left(B+\left(\begin{array}{cc}
0 & 1 \\
-1 & 0
\end{array}\right)\right)\right] e^{i \Theta B} .
$$


Note that

$$
\left(\begin{array}{c}
u_{n} \\
u_{n+1}
\end{array}\right)=B_{n} \cdot B_{n-1} \cdots B_{2}\left(\begin{array}{c}
u_{1} \\
u_{2}
\end{array}\right)
$$

is uniformly bounded, iff $\tilde{B}_{n} \cdot \tilde{B}_{n-1} \cdots \tilde{B}_{2} U W^{-1}\left(\begin{array}{c}u_{1} \\ u_{2}\end{array}\right)$ is uniformly bounded.

\section{Absolutely continuous spectrum of $J$}

As we mentioned in the Introduction the methods used in the analysis of the boundedness of solutions of (1.1) consist in repeated use of the Harris-Lutz (HL) transform and Kiselev's lemma as well.

We shall analyse the case $\beta=\alpha$, i.e. situation (a) mentioned above.

Define the product

$$
Z_{n}=e^{i \varphi_{n} B}\left(I+\Gamma_{n}\right)\left(I+\Omega_{n}\right),
$$

where $\varphi_{n} \in \mathbb{R}, \Omega_{n}, \Gamma_{n}$ are suitable $2 \times 2$ matrices of small norm. We shall describe precisely the choice of parameters $\varphi_{n}, \Omega_{n}, \Gamma_{n}$ below.

Take $N_{0}$ so large that $Z_{k}$ are invertible for all $k \geq N_{0}$. Consider the identity

$$
\tilde{B}_{n} \cdots \tilde{B}_{2}=Z_{n+1}\left(Z_{n+1}^{-1} \tilde{B}_{n} Z_{n}\right) \cdots\left(Z_{N_{0}+1}^{-1} \tilde{B}_{N_{0}} Z_{N_{0}}\right) Z_{N_{0}}^{-1} \tilde{B}_{N_{0}-1} \cdots \tilde{B}_{2} .
$$

We concentrate on the factor $Z_{n+1}^{-1} \tilde{B}_{n} Z_{n}$.

We proceed step by step by constructing $\varphi_{n}, \Gamma_{n}$ and $\Omega_{n}$.

Step 1 (the choice of $\varphi_{n}$ ). Put $\varphi_{n}=n \Theta$ and note that

$$
e^{-i \varphi_{n+1} B}\left(\begin{array}{ll}
0 & 1 \\
1 & 0
\end{array}\right) e^{i \varphi_{n+1} B}=\left(\begin{array}{cc}
0 & \exp (-2 i(n+1) \Theta) \\
\exp (2 i(n+1) \Theta) & 0
\end{array}\right)
$$

and

$$
e^{-i \varphi_{n+1} B}\left(\begin{array}{cc}
0 & 1 \\
-1 & 0
\end{array}\right) e^{i \varphi_{n+1} B}=\left(\begin{array}{cc}
0 & \exp (-2 i(n+1) \Theta) \\
-\exp (2 i(n+1) \Theta) & 0
\end{array}\right) .
$$

Hence (using (2.9)) we have

$$
\begin{gathered}
e^{-i \varphi_{n+1} B} \tilde{B}_{n} e^{i \varphi_{n} B}=\left(1+\frac{\varepsilon_{n}}{2}\right) I-\frac{\varepsilon_{n}}{2}\left(\begin{array}{cc}
0 & \exp (-2 i(n+1) \Theta) \\
\exp (2 i(n+1) \Theta) & 0
\end{array}\right) \\
+\frac{i \eta_{n}}{2} \operatorname{ctg} \Theta\left[B+\left(\begin{array}{cc}
0 & \exp (-2 i(n+1) \Theta) \\
-\exp (2 i(n+1) \Theta) & 0
\end{array}\right)\right] .
\end{gathered}
$$

Step 2 (the choice of $\Gamma_{n}$ ). In this step we assume that $\gamma>3 / 4$ and $\alpha+\gamma>3 / 2$.

Denote

$$
\begin{gathered}
Q_{n}:=\left(\begin{array}{cc}
0 & \exp (-2 i(n+1) \Theta) \\
\exp (2 i(n+1) \Theta) & 0
\end{array}\right), \\
0 \quad \exp (-2 i(n+1) \Theta) \\
T_{n}:=\left(\begin{array}{c}
0 \\
-\exp (2 i(n+1) \Theta)
\end{array}\right), \rho_{n}:=\frac{i \eta_{n}}{2} \operatorname{ctg} \Theta .
\end{gathered}
$$

Now, according to a slightly modified Harris-Lutz Ansatz we look for $\Gamma_{n}$ in the form $\left(\begin{array}{cc}0 & a_{n} \\ -\bar{a}_{n} & 0\end{array}\right)$. 
Since $\left(I+\Gamma_{n+1}\right)^{-1}=\left(1+\left|a_{n+1}\right|^{2}\right)^{-1}\left(I-\Gamma_{n+1}\right)$ we have

$$
\begin{gathered}
\left(I+\Gamma_{n+1}\right)^{-1}\left[\left(1+\frac{\varepsilon_{n}}{2}\right) I-\frac{\varepsilon_{n}}{2} Q_{n}+\rho_{n} B+\rho_{n} T_{n}\right]\left(I+\Gamma_{n}\right) \\
=\left(1+\left|a_{n+1}\right|^{2}\right)^{-1}\left[\left(1+\frac{\varepsilon_{n}}{2}\right) I-\frac{\varepsilon_{n}}{2} Q_{n}+\rho_{n} B+\rho_{n} T_{n}\right. \\
-\left(1+\frac{\varepsilon_{n}}{2}\right) \Gamma_{n+1}+\frac{\varepsilon_{n}}{2} \Gamma_{n+1} Q_{n}-\rho_{n} \Gamma_{n+1} B \\
-\rho_{n} \Gamma_{n+1} T_{n}+\left(1+\frac{\varepsilon_{n}}{2}\right) \Gamma_{n}-\frac{\varepsilon_{n}}{2} Q_{n} \Gamma_{n}+\rho_{n} B \Gamma_{n} \\
+\rho_{n} T_{n} \Gamma_{n}-\left(1+\frac{\varepsilon_{n}}{2}\right) \Gamma_{n+1} \Gamma_{n}+\frac{\varepsilon_{n}}{2} \Gamma_{n+1} Q_{n} \Gamma_{n} \\
\left.-\rho_{n} \Gamma_{n+1} B \Gamma_{n}-\rho_{n} \Gamma_{n+1} T_{n} \Gamma_{n}\right] .
\end{gathered}
$$

Now, choose $\Gamma_{n}$ to satisfy

$$
\left(1+\frac{\varepsilon_{n}}{2}\right)\left(\Gamma_{n}-\Gamma_{n+1}\right)=-\rho_{n} T_{n} .
$$

It follows that

$$
\Gamma_{n}=-\sum_{k=n}^{\infty} \rho_{k}\left(1+\frac{\varepsilon_{k}}{2}\right)^{-1} T_{k} .
$$

Corollary 2.2 ensures that

$$
\left\|\Gamma_{n}\right\|=O\left(n^{\frac{1}{2}-\gamma+\omega}\right), \quad \text { for almost all } \Theta \in(-\pi, \pi),
$$

where $\omega>0$ is arbitrary small.

Since $\left(1+\frac{\varepsilon_{n}}{2}\right) \Gamma_{n+1} \Gamma_{n}=-\left|a_{n+1}\right|^{2}\left(1+\frac{\varepsilon_{n}}{2}\right) I-\rho_{n} \Gamma_{n+1} T_{n}$, equalities (3.1), (3.2) and our assumptions $\alpha+\gamma>3 / 2, \gamma>3 / 4$ imply

$$
\begin{gathered}
\left(I+\Gamma_{n+1}\right)^{-1} e^{-i \varphi_{n+1} B} \tilde{B}_{n} e^{i \varphi_{n} B}\left(I+\Gamma_{n}\right) \\
=\left(1+\frac{\varepsilon_{n}}{2}\right) I-\frac{\varepsilon_{n}}{2}\left(1+\left|a_{n+1}\right|^{2}\right)^{-1} Q_{n}+\rho_{n}\left(1+\left|a_{n+1}\right|^{2}\right)^{-1} B+R_{n},
\end{gathered}
$$

where $\left\{\left\|R_{n}\right\|\right\} \in l^{1}$.

Indeed, the norms of matrices $\rho_{n} B \Gamma_{n}$ and $\varepsilon_{n} \Gamma_{n+1} Q_{n}$ are of order $O\left(n^{\frac{1}{2}-2 \gamma+\omega}\right)$ and $O\left(n^{\frac{1}{2}-\alpha-\gamma+\omega}\right)$, respectively. Here $\omega>0$ is arbitrary small. The other matrices which have been included in the $R_{n}$ term have the same or higher orders of decay.

But $\varepsilon_{n}\left|a_{n+1}\right|^{2}=O\left(n^{1-\alpha-2 \gamma+2 \omega}\right)$ and $\rho_{n}\left|a_{n+1}\right|^{2}=O\left(n^{1-3 \gamma+2 \omega}\right), \omega>0$, and the right hand side of (3.3) can be written as

$$
\left(1+\frac{\varepsilon_{n}}{2}\right)\left[I-\frac{\varepsilon_{n}}{2} Q_{n}+\rho_{n} B+\tilde{R}_{n}\right]
$$

where $\left\{\left\|\tilde{R}_{n}\right\|\right\} \in l^{1}$.

Step 3 (the choice of $\Omega_{n}$ ). In this step we assume that $\alpha>2 / 3$.

Let $\Omega_{n}=\left(\begin{array}{cc}0 & b_{n} \\ \bar{b}_{n} & 0\end{array}\right)$ and denote $\tilde{\varepsilon}_{n}:=\frac{\varepsilon_{n}}{2}$,

Since $\left(I+\Omega_{n+1}\right)^{-1}=\left(1-\left|b_{n+1}\right|^{2}\right)^{-1}\left(I-\Omega_{n+1}\right)$ we have

$$
\begin{gathered}
\left(I+\Omega_{n+1}\right)^{-1}\left[I-\tilde{\varepsilon}_{n} Q_{n}+\rho_{n} B\right]\left(I+\Omega_{n}\right) \\
=\left(1-\left|b_{n+1}\right|^{2}\right)^{-1}\left[I-\tilde{\varepsilon}_{n} Q_{n}+\rho_{n} B-\Omega_{n+1}\right. \\
+\tilde{\varepsilon}_{n} \Omega_{n+1} Q_{n}-\rho_{n} \Omega_{n+1} B+\Omega_{n}-\tilde{\varepsilon}_{n} Q_{n} \Omega_{n} \\
\left.+\rho_{n} B \Omega_{n}-\Omega_{n+1} \Omega_{n}+\tilde{\varepsilon}_{n} \Omega_{n+1} Q_{n} \Omega_{n}-\rho_{n} \Omega_{n+1} B \Omega_{n}\right] .
\end{gathered}
$$


Define $\Omega_{n}$ by the relation

$$
\Omega_{n}-\Omega_{n+1}=\tilde{\varepsilon}_{n} Q_{n}
$$

Thus

$$
\Omega_{n}=\sum_{k=n}^{\infty} \tilde{\varepsilon}_{k} Q_{k}
$$

Again by Corollary 2.2 we know that

$$
\left\|\Omega_{n}\right\|=O\left(n^{1 / 2-\alpha+\omega}\right), \omega>0, \quad \text { a.e. in } \Theta \in(-\pi, \pi) .
$$

Similarly as above using (3.6) and the assumption $\alpha>2 / 3$ we can write the right hand side of (3.5) as

$$
\left(1-\left|b_{n+1}\right|^{2}\right)^{-1}\left[I+\rho_{n} B-\tilde{\varepsilon}_{n}\left(Q_{n} \Omega_{n}-\Omega_{n+1} Q_{n}\right)-\Omega_{n+1} \Omega_{n}+H_{n}\right],
$$

where $\left\{\left\|H_{n}\right\|\right\} \in l^{1}$. This can be easily checked by observing that matrices $\rho_{n} \Omega_{n+1} B$ have norms of order $O\left(n^{\frac{1}{2}-\alpha-\gamma+\omega}\right), \omega>0$. The other matrices absorbed in the $H_{n}$ term have the same or higher order estimations of their norms.

But $\Omega_{n+1} \Omega_{n}=\Omega_{n+1}^{2}+\tilde{\varepsilon}_{n} \Omega_{n+1} Q_{n}$ and the right hand side of (3.7) equals

$$
\begin{gathered}
I+\left(1-\left|b_{n+1}\right|^{2}\right)^{-1}\left[\rho_{n} B-\tilde{\varepsilon}_{n} Q_{n} \Omega_{n}+H_{n}\right] \\
=I+\rho_{n} B-\tilde{\varepsilon}_{n} Q_{n} \Omega_{n}+\tilde{H}_{n}, \quad \text { where }\left\{\left\|\tilde{H}_{n}\right\|\right\} \in l^{1}
\end{gathered}
$$

by using the estimation $\left|b_{n}\right|=O\left(n^{1 / 2-\alpha+\omega}\right)$ a.e. in $\Theta \in(-\pi, \pi)$.

Therefore the problem of boundedness of the solution $\left\{u_{n}\right\}$ of (1.1) has been reduced to the question of uniform boundedness from above and below (in a proper way) of the matrix product

$$
\prod_{k=2}^{n}\left(I+\rho_{n} B-\tilde{\varepsilon}_{n} Q_{n} \Omega_{n}\right)
$$

This is immediate consequence of Lemma 2.3.

Here we meet new obstacles in contrast to the DSO case. This is one situation where the discrete string operator displays its character.

Note that the matrix $\rho_{n} B-\tilde{\varepsilon}_{n} Q_{n} \Omega_{n}$ is of diagonal form given by

where $z_{n}=\exp (-2 i(n+1) \Theta)$.

$$
\left(\begin{array}{cc}
\rho_{n}-\tilde{\varepsilon}_{n} z_{n} \bar{b}_{n} & 0 \\
0 & \bar{\rho}_{n}-\tilde{\varepsilon}_{n} \bar{z}_{n} b_{n}
\end{array}\right)
$$

Due to the above constructions and definition of $B_{n}$ we have (see (3.4))

$$
\begin{gathered}
\frac{\lambda_{1}}{\lambda_{n}}=\operatorname{det}\left(\tilde{B}_{n} \cdots \tilde{B}_{2}\right)=\operatorname{det}\left(I+\Gamma_{n+1}\right) . \\
\prod_{k=2}^{n}\left(1+\tilde{\varepsilon}_{k}\right) \operatorname{det} \prod_{k=2}^{n}\left(I-\tilde{\varepsilon}_{k} Q_{k}+\rho_{k} B+\tilde{R}_{k}\right) \cdot \operatorname{det}\left(I+\Gamma_{2}\right)^{-1} .
\end{gathered}
$$

Combining (3.4) and (3.8) we have

$$
\begin{gathered}
I-\tilde{\varepsilon}_{n} Q_{n}+\rho_{n} B+\tilde{R}_{n} \\
=\left(I+\Omega_{n+1}\right)\left(I+\rho_{n} B-\tilde{\varepsilon}_{n} Q_{n} \Omega_{n}+\tilde{H}_{n}\right)\left(I+\Omega_{n}\right)^{-1}+\tilde{R}_{n} \\
=I+\rho_{n} B-\tilde{\varepsilon}_{n} Q_{n} \Omega_{n}+\tilde{\tilde{H}}_{n}, \quad \text { where }\left\{\left\|\tilde{\tilde{H}}_{n}\right\|\right\} \in l^{1} .
\end{gathered}
$$

Since $\left\{\varepsilon_{k}\right\} \in l^{2}$ and $\sum_{k} \varepsilon_{k}$ is convergent, the product $\prod_{k=2}^{n}\left(1+\tilde{\varepsilon}_{k}\right)$ converges also. 
Therefore (3.9) and the above equalities imply that

$$
\operatorname{det} \prod_{k=N_{o}}^{n}\left(I+\rho_{k} B-\tilde{\varepsilon}_{k} Q_{k} \Omega_{k}+\tilde{\tilde{H}}_{k}\right)
$$

is convergent. Hence

$$
\operatorname{det} \prod_{k=N_{o}}^{n}\left(I+\rho_{k} B-\varepsilon_{k} Q_{k} \Omega_{k}\right)=\prod_{k=N_{o}}^{n}\left|1+\rho_{k}-\tilde{\varepsilon}_{k} z_{k} \bar{b}_{k}\right|^{2}
$$

is convergent!

It follows (remember that $\rho_{k}$ and $\varepsilon_{k}$ belong to $l^{2}$ ) that the series $\sum_{k} \tilde{\varepsilon}_{k} \operatorname{Re}\left(z_{k} \bar{b}_{k}\right)$ is convergent (because $\operatorname{Re} \rho_{k}=0$ ). Finally we infer (by Lemma 2.3) that the matrix product $\prod_{k=N_{o}}^{n}\left(I+\rho_{k} B-\tilde{\varepsilon}_{k} Q_{k} \Omega_{k}\right)$ is uniformly bounded from below and above.

This proves the following

Theorem 3.1. Let $\varepsilon_{n}=O\left(n^{-\alpha}\right), \eta_{n}=O\left(n^{-\gamma}\right)$, where $\alpha$ and $\gamma$ satisfy the following inequalities: $\gamma \geq \alpha>2 / 3, \gamma>3 / 4$ and $\alpha+\gamma>3 / 2$. Then the absolutely continuous component of the spectrum of $J$ fills the interval $(-2,2)$.

Hence Theorem 3.1 exhibits an interesting interplay $(\alpha+\gamma>3 / 2)$ between parameter $\alpha$ (which measures local behaviour of $\lambda_{k}$ ) and $\gamma$ (related to the local oscillation property of $\lambda_{k}$ ); see (2.2) and (2.4). By analysing the proof of Theorem 3.1 we can even obtain the asymptotics of the solution $\left\{u_{n}\right\}$ of (1.1).

Indeed, transforming back via (3.8), (3.7) and (3.3) we have

$$
\left(\begin{array}{c}
u_{n} \\
u_{n+1}
\end{array}\right)=W U^{-1} Z_{n+1} \tilde{\tilde{B}}_{n} \cdots \tilde{\tilde{B}}_{N_{0}} Z_{N_{0}}^{-1} \tilde{B}_{N_{0}-1} \cdots \tilde{B}_{2} U W^{-1}\left(\begin{array}{c}
u_{1} \\
u_{2}
\end{array}\right) .
$$

where $\tilde{B}_{k}=Z_{k+1}^{-1} \tilde{B}_{k} Z_{k}$. Again by (3.8) we know that for $k \geq N_{0}$

$$
\tilde{\tilde{B}}_{k}=I+\rho_{k} B-\tilde{\varepsilon}_{k} Q_{k} \Omega_{k}+\tilde{H}_{k}, \quad \text { where }\left\{\left\|\tilde{H}_{k}\right\|\right\} \in l^{1}
$$

and $I+\rho_{k} B-\tilde{\varepsilon}_{k} Q_{k} \Omega_{k}$ is the diagonal matrix given by

$$
\left(\begin{array}{cc}
1+\rho_{k}-\tilde{\varepsilon}_{k} z_{k} \bar{b}_{k} & 0 \\
0 & 1+\bar{\rho}_{k}-\tilde{\varepsilon}_{k} \bar{z}_{k} b_{k}
\end{array}\right) .
$$

Now formula (3.10) implies that (for a suitable choice of $u_{1}$ and $u_{2}$ )

$$
u_{n}=e^{i n \Theta} \prod_{k=N_{0}}^{n}\left(1+\rho_{k}-\tilde{\varepsilon}_{k} z_{k} \bar{b}_{k}\right)\left(d+c_{n}\right), \quad d \neq 0
$$

where $c_{n}=o(1)$ as $n$ tends to infinity. This equality can be checked using the forms of matrices $W U^{-1}, Z_{n+1}$ and the uniform boundedness of

$$
\prod_{k=N_{0}}^{n}\left(1+\rho_{k}-\tilde{\varepsilon}_{k} z_{k} \bar{b}_{k}\right)
$$

Since $\sum_{k} \varepsilon_{k} \Re\left(\bar{z}_{k} b_{k}\right)$ is a.e. convergent, we can rewrite the above equality (after a 
renormalization of $u_{n}$ ) as

$$
\begin{gathered}
u_{n}=e^{i n \Theta} \exp \sum_{k=N_{0}}^{n}\left(\rho_{k}-i \tilde{\varepsilon}_{k} \Im\left(z_{k} \bar{b}_{k}\right)\right) \\
\cdot \exp \left[-\sum_{k=N_{0}}^{n} \tilde{\varepsilon}_{k} \Re\left(z_{k} \bar{b}_{k}\right)\right]\left(1+\tilde{c}_{n}\right),
\end{gathered}
$$

where $\tilde{c}_{n}=o(1)$ as $n \rightarrow \infty$.

Summing up, we have

Theorem 3.2. If the weights $\left\{\lambda_{k}\right\}$ satisfy all assumptions of Theorem 3.1, then we have two linearly independent solutions $\{u\},\{\bar{u}\}$ of the equation (1.1) with the asymptotics given for almost all $\lambda \in(-2,2)$ by

$$
u_{n}=e^{i n \Theta} \exp \left\{\frac{i}{2} \sum_{k=2}^{n}\left[\left(2 \delta_{k} \operatorname{ctg} \Theta-\varepsilon_{k} \sum_{s=k}^{\infty} \frac{\varepsilon_{s}}{2} \sin 2(s-k) \Theta\right]\right\}(1+o(1)),\right.
$$

where $\lambda=2 \cos \Theta$.

Formula (3.12) shows that asymptotics of the solution $u$ contain besides the WKB factor

$$
\exp i\left[n \Theta+\operatorname{ctg} \Theta \sum_{k=2}^{n} \delta_{k}\right]
$$

the additional term

$$
\exp \frac{i}{2}\left[-\sum_{k=2}^{n} \varepsilon_{k} \sum_{s=k}^{\infty} \frac{\varepsilon_{s}}{2} \sin (s-k) \Theta\right]
$$

which has many more complicated characters in comparison with the first one. On the other hand it is remarkable that the non-oscillating factor

$$
\exp \left(\sum_{k=2}^{n} \varepsilon_{k} \Re\left(b_{k} \bar{z}_{k}\right)\right)
$$

disappears due to the a.e. convergence of the series $\sum_{k} \varepsilon_{k} \Re\left(b_{k} \bar{z}_{k}\right)$. Again we can observe an interesting feature of the discrete string operator.

Despite the above theorem we have no information concerning the set in $\mathbb{R}_{+}$on which the singular part of the spectral measure might be supported. In our earlier paper [7] some sufficient conditions were found implying the absence of the point spectrum of $J$ in $(-2,2)$, however.

Remark 3.3. Recently Kiselev [10] found a beautiful new technique for dealing with absolute continuity of the Discrete Schrödinger Operator

$$
u_{n} \rightarrow u_{n+1}+u_{n-1}+V(n) u_{n}, \quad \text { provided } V(n)=O\left(n^{-2 / 3-\varepsilon}\right), \varepsilon>0 .
$$

His method combined with our approach looks promising to allow us to extend results of the present paper to a more general class of perturbation. 


\section{REFERENCES}

[1] Yu.M. Berezanskii, Expansions in Eigenfunctions of Selfadjoint Operators, Naukova Dumka, Kiev, (1965) (in Russian). MR 36:5769

[2] H. Behncke, Absolute continuity of Hamiltonians with von Neumann Wigner Potentials II, Manuscripta Math. 71, (1991) 163-189. MR 93f:81031

[3] E.A. Coddington and N. Levinson, Theory of Ordinary Differential Equations, McGraw-Hill, New York, 1955. MR 16:1022b

[4] J. Dombrowski, Cyclic operators, commutators, and absolutely continuous measures, Proc. Amer. Math. Soc., Vol 100, No 3, (1987) 457-462. MR 88i:47014

[5] D.J. Gilbert and D.B. Pearson, On subordinacy and analysis of the spectrum of onedimensional Schrödinger operators, J.Math. Anal. Appl. 128 (1987), 30-56. MR 89a:34033

[6] W.A. Harris and D.A. Lutz, Asymptotic integration of adiabatic oscillators, J. Math. Anal. Appl. 51 (1975), 76-93. MR 51:6069

[7] J. Janas and S.N. Naboko, On the point spectrum of some Jacobi matrices, JOT, to appear.

[8] S. Khan and D.B. Pearson, Subordinacy and spectral theory for infinite matrices, Helv. Phys. Acta 65 (1992) 505-527. MR 94a:47066

[9] A. Kiselev, Absolute continuous spectrum of one-dimensional Schrödinger operators and Jacobi matrices with slowly descreasing potentials, Comm. Math. Phys. 179 (1996), 377-400.

[10] _ Preservation of the absolutely continuous spectrum of Schrödinger equation under perturbations by slowly decreasing potentials and a.e. convergence of integral operators (1997) (preprint).

[11] G. Stolz, Bounded solutions and absolute continuity of Sturm-Liouville operators, J. Math. Anal. Appl. 169 (1992) 210-228. MR 93f:34141

[12] _ Spectral Theory for Slowly Oscillating Potentials I. Jacobi Matrices. Manuscripta Math. 84 (1994) 245-260. MR 95k:47050

[13] J. Weidmann, Uniform Nonsubordinacy and the Absolutely Continuous Spectrum, Analysis 16 (1996) 89-99. MR 96m:34154

Institute of Mathematics, Polish Academy of Sciences, Cracow Branch, Sw. Tomasza 30, 31-027 Krakow, Poland

E-mail address: najanas@cyf-kr.edu.pl

Department of Mathematical Physics, Institute for Physics, St. Petersburg UniverSity, Ulianovskaia 1, 198904, St. Petergoff, Russia

E-mail address: naboko@snoopy.phys.spbu.ru 